\title{
Residual Symmetry, Bäcklund Transformation, and Soliton Solutions of the Higher-Order Broer-Kaup System
}

\author{
Yarong Xia, ${ }^{1,2}$ Ruoxia Yao $\mathbb{D}^{1},{ }^{1}$ and Xiangpeng Xin $\mathbb{D}^{3}$ \\ ${ }^{1}$ School of Computer Science, Shaanxi Normal University, Xi'an 710062, China \\ ${ }^{2}$ School of Information and Engineering, Xi'an University, Xi'an 710065, China \\ ${ }^{3}$ School of Mathematical Sciences, Liaocheng University, Liaocheng 252029, China \\ Correspondence should be addressed to Ruoxia Yao; rxyao@snnu.edu.cn and Xiangpeng Xin; xinxiangpeng@lcu.edu.cn
}

Received 7 March 2021; Revised 19 April 2021; Accepted 26 April 2021; Published 19 May 2021

Academic Editor: Wen-Xiu Ma

Copyright (c) 2021 Yarong Xia et al. This is an open access article distributed under the Creative Commons Attribution License, which permits unrestricted use, distribution, and reproduction in any medium, provided the original work is properly cited.

Under investigation in this paper is the higher-order Broer-Kaup(HBK) system, which describes the bidirectional propagation of long waves in shallow water. Via the standard truncated Painleve expansion method, the residual symmetry of this system is derived. By introducing an appropriate auxiliary-dependent variable, the residual symmetry is successfully localized to Lie point symmetries. Via solving the initial value problems, the finite symmetry transformations are presented. However, the solution which obtained from the residual symmetry is a special group invariant solutions. In order to find more general solution of HBK system, we further generalize the residual symmetry method to the consistent tanh expansion (CTE) method and prove that the HBK system is CTE solvable, then the resonant soliton solutions and interaction solutions among different nonlinear excitations are obtained by the CET method.

\section{Introduction}

Since the Lie group theory was proposed by Sophus Lie to study differential equation, the symmetry theory has been widely developed to study nonlinear equation. As is known, thanks to the classical or nonclassical Lie group method [1, 2], Lie point symmetries of a differential system can be obtained, from which one can reduce the dimensions of partial differential equations and proceed to construct group invariant solutions by similarity reductions [3-8]. Nevertheless, for the integrable system, there may exist nonlocal symmetries which are related to inverse recursion operators [9, 10], Darboux transformation(DT) [11], Bäcklund transformation(BT) [12-15], conformal invariance [16], negative hierarchies $[17,18]$, and so on. Recently, it is found that Painlevé analysis can also be applied to obtain nonlocal symmetries, and this type of nonlocal symmetries corresponds to the residues with respect to the singular manifold of the truncated Painlevé expansion, which are also called residual symmetries [19-23].
One knows that the nonlocal symmetries cannot be used to construct explicit solutions to differential equations directly; naturally, we need to transform the nonlocal symmetries into local ones by introducing suitable prolonged systems. In reference [24, 25], the authors has considered the localizations of the residual symmetries and found related finite transformation. Furthermore, by developing the truncated Painlevé expansion, Lou introduced the definition of consistent Riccati expansion solvable [26], and this method is greatly valid for constructing both possible new integrable systems and interaction solutions between a soliton and other types of nonlinear excitations. A consistent tanh expansion is a special simplified form of the consistent Riccati expansion defined in literature [27]. So, through the relationship of the nonlocal symmetry and the consistent Riccati expansion solvable or the consistent tanh expansion solvable, one can construct the solutions of nonlinear evolution equations. On account of this, there are a lot of papers here to study this problem [28-32]. 
This paper concentrates on investigating the following higher-order Broer-Kaup system

$$
\begin{gathered}
u_{t}+4\left(u_{x x}+u^{3}+6 u v-3 u u_{x}\right)_{x}=0 \\
v_{t}+4\left(v_{x x}+3 u v_{x}+3 u^{2} v+3 v^{2}\right)_{x}=0
\end{gathered}
$$

which was first derived and studied by Lou and $\mathrm{Hu}$ [33]. The system (1) can be seen as an extension of the known BroerKaup system which is often used to model the bidirectional propagation of long waves in shallow water. Some special solutions to the system (1) were given in reference [34], huang [35] studied the explicit $N$-fold Darboux transformation and multisoliton solutions to the system (1), Li et al. [36] investigated the Painlevé analysis and new analytic solutions to the system (1), and the interaction solutions to the system (1) were derived in reference [37]. However, the residual symmetries, the consistent tanh expansion solvable, and interaction solutions of the higher-order Broer-Kaup system have not yet been studied, which is the prime objective of this paper.

\section{The Residual Symmetry of the Higher-Order Broer-Kaup System and Its Localization}

The higher-order Broer-Kaup system is Painlevé integrable, and its truncated Painlevé expansion can be expressed as

$$
u=u_{0}+\frac{u_{1}}{\phi}, v=v_{0}+\frac{v_{1}}{\phi}+\frac{v_{2}}{\phi^{2}}
$$

where $u_{0}, u_{1}, v_{0}, v_{1}, v_{2}$ are functions of $x$ and $t$, and $\phi(x, t)=0$ is the singular manifold equation. Substituting equation (2) into equation (1), then, collecting the power of $\phi$ and making its coefficients equal to zero, we get two systems of $\phi$, and the concert expressions are in the appendix.

Vanishing the coefficients of $1 / \phi^{4}$ in the first one of system (1) and the coefficients of $1 / \phi^{5}$ in the second one of system in the appendix, we have

$$
u_{1}=\phi_{x}, v_{2}=-\phi_{x}^{2} .
$$

Vanishing the coefficients of $1 / \phi^{3}$ in the first one of the system (1) and the coefficients of $1 / \phi^{4}$ in the second system in the appendix and using (3) leads to

$$
v_{1}=\phi_{x x}
$$

Vanishing the coefficients of $1 / \phi^{2}$ in the first one of the system (1) and the coefficients of $1 / \phi^{3}$ in the second system in the appendix, then, by using (3), (4) leads to

$\phi_{t}=-12 \phi_{x} u_{0}^{2}-12 \phi_{x x} u_{0}-24 \phi_{x} v_{0}+12 \phi_{x} u_{0, x}-4 \phi_{x x x}, v_{0}=u_{0, x}$,

using equations (3), (4), and (5), it is not difficult to find that the coefficient of $1 / \phi^{2}$ in the second system of appendix identically becomes zero. The coefficients of $\phi^{0}$ in appendix are nothing but the higher-order Broer-Kaup system (1) for $u$ $=u_{0}, v=v_{0}$, while the coefficient of $1 / \phi$ in appendix is just the symmetry equation for system (1) with the symmetry

$$
\sigma^{u_{0}}=u_{1}, \sigma^{v_{0}}=v_{1} .
$$

That is to say, when $u_{0}, v_{0}$ are the solutions of system (1), $u_{1}, v_{1}$ are the solutions to the corresponding symmetry equation. From above analysis and the standard truncated Painlevé expansion (2), the following Bäcklund transformation theorem and residual symmetry theorem arise.

Theorem 1. If the function $\phi$ satisfies equation (5), then

$$
\begin{aligned}
& u=u_{0}+\frac{\phi_{x}}{\phi}=u_{0}+(\ln \phi)_{x} \\
& v=v_{0}+\frac{\phi_{x x}}{\phi}-\frac{\phi_{x}^{2}}{\phi^{2}}=v_{0}+(\ln \phi)_{x x}
\end{aligned}
$$

are the Bäcklund transformation between solutions $\left\{u_{0}, v_{0}\right\}$ and $\{u, v\}$.

Theorem 2. The higher-order Broer-Kaup system (1) has the following residual symmetry

$$
\sigma_{u}=\phi_{x}, \sigma_{v}=\phi_{x x}
$$

where $u, v$, and $\phi$ satisfy the Bäcklund transformation (7).

Proof. From the coefficients of $1 / \phi$ in appendix, we find that $u_{1}=\phi_{x}, v_{1}=\phi_{x x}$ is just the solution of the symmetry equation for the system (1), where $u_{1}, v_{1}$ are just the residual of truncated Painlevé expansion (2) when $u=u_{0}, v=v_{0}$ are the solution of system (1); so based on the definition of residual symmetry, the nonlocal symmetry (8) is also called residual symmetry.

Since $u_{1}$ and $v_{1}$ are nonlocal symmetries, as we all know, the nonlocal symmetries cannot construct explicit solutions to differential equations directly. One can naturally believe that we need to transform the nonlocal symmetries into local ones. To this end, we introduce new variables $f$ and $g$ to eliminate the space derivatives of $\phi$ by

$$
f=\phi_{x}, g=f_{x}
$$

then the nonlocal symmetry of the higher-order Broer-Kaup system (1) is localized to the following Lie point symmetries

$$
\sigma_{u}=f, \sigma_{v}=g, \sigma_{\phi}=-\phi^{2}, \sigma_{f}=-2 \phi f, \sigma_{g}=-2 f^{2}-2 \phi g,
$$

for the prolonged systems (1), (7), and (9) with the Lie point symmetry vector

$$
V=f \partial_{u}-g \partial_{v}-\phi^{2} \partial_{\phi}-2 \phi f \partial_{f}-2\left(f^{2}+\phi g\right) \partial_{g}
$$

To proceed, we study the finite symmetry transformation 
of Lie point symmetries (10). According to Lie's first theorem, by solving the following initial value problems,

$$
\begin{aligned}
& \frac{\mathrm{d} \widehat{u}(\varepsilon)}{\mathrm{d} \varepsilon}=\widehat{f}(\varepsilon), \widehat{u}(0)=u, \\
& \frac{\mathrm{d} \widehat{v}(\varepsilon)}{\mathrm{d} \varepsilon}=\widehat{g}(\varepsilon), \widehat{v}(0)=v, \\
& \frac{\mathrm{d} \widehat{f}(\varepsilon)}{\mathrm{d} \varepsilon}=-2 \widehat{f}(\varepsilon) \widehat{\phi}(\varepsilon), \widehat{f}(0)=f, \\
& \frac{\mathrm{d} \widehat{g}(\varepsilon)}{\mathrm{d} \varepsilon}=-2 f \wedge^{2}(\varepsilon)-2 \widehat{\phi}(\varepsilon) \widehat{g}(\varepsilon), \widehat{g}(0)=g \\
& \frac{\mathrm{d} \widehat{\phi}(\varepsilon)}{\mathrm{d} \varepsilon}=-\phi \wedge^{2}(\varepsilon), \widehat{\phi}(0)=\phi
\end{aligned}
$$

one can easily obtain the symmetry group transformation theorem as follows:

Theorem 3. If $\{u, v, \phi, f, g\}$ is a solution to the prolonged system (1), (7), and (9), then so $\{\widehat{u}, \widehat{v}, \widehat{\phi}, \widehat{f}, \widehat{g}\}$ is given by

$$
\begin{aligned}
& \widehat{u}=-\frac{f}{(\varepsilon \phi+1) \phi}+\frac{u \phi+f}{\phi}, \widehat{\phi}=\frac{\phi}{\varepsilon \phi+1}, \widehat{f}=\frac{f}{(\varepsilon \phi+1)^{2}}, \\
& \widehat{v}=\frac{\left(v \phi^{2}+g \phi-f^{2}\right) \varepsilon^{2}+(2 \phi v+g) \varepsilon+v}{(1+\varepsilon \phi)^{2}}, \\
& \widehat{g}=\frac{g}{(\varepsilon \phi+1)^{2}}-\frac{2 f^{2} \varepsilon}{(\varepsilon \phi+1)^{3}} .
\end{aligned}
$$

Remark 4. From Theorem 3, we find an interesting phenomenon that the residual symmetry $\left\{u_{1}=\phi_{x}, v_{1}=\phi_{x x}\right\}$ coming from the truncated Painlevé expansion is just the infinitesimal form of the group. Actually, the above group transformation is equivalent to the truncated Painlevé expansion (2) and (3) since the singularity manifold equations (1), (7), and (9) are form invariant under the transformation

$$
1+\varepsilon \phi \rightarrow \phi \text { with }\left(\varepsilon f \rightarrow \phi_{x}, \varepsilon g \rightarrow \phi_{x x}\right)
$$

Remark 5. For given solutions $u, v$ of (1), through the finite symmetry transformation (13) will arrive new solutions $\widehat{u}, \widehat{v}$ . For example, we take a simple solutions as $u=0, v=0$ for (1), and then from equation (5), through solving an ordinary differential equation, we get $\phi$; further, by equation (9), we get the expression of $f$, and the concert form of $\phi$ and $f$ is as follows:

$$
\begin{aligned}
\phi= & \left(C_{1} \exp \left(\frac{-1}{2} b_{1}^{1 / 3}(i \sqrt{3}+1) x\right)+C_{2} \exp \right. \\
& \left.\cdot\left(\frac{1}{2} b_{1}^{1 / 3}(i \sqrt{3}-1) x\right)+C_{3} b_{1}^{1 / 3} \exp (\sqrt{3} x)\right) C_{4} \exp \left(-4 b_{1} t\right),
\end{aligned}
$$

$$
\begin{aligned}
f= & \left(\frac{-1}{2} C_{1} b_{1}^{1 / 3}(i \sqrt{3}+1) \exp \left(\frac{-1}{2} b_{1}^{1 / 3}(i \sqrt{3}+1) x\right)\right. \\
& +\frac{1}{2} C_{2} b_{1}^{1 / 3}(i \sqrt{3}-1) x \exp \left(\frac{1}{2} b_{1}^{1 / 3}(i \sqrt{3}-1) x\right) \\
& \left.+C_{3} b_{1}^{1 / 3} \exp (\sqrt{3} x)\right) C_{4} \exp \left(-4 b_{1} t\right)
\end{aligned}
$$

then, by help of equation (13), we can get a pair of new solution $\widehat{u}$ and $\widehat{v}$ (the concrete expressions are omitted here since of its prolixity), where $C_{i},(i=1,2,3,4)$ and $b_{1}$ are arbitrary constants.

However, the solution obtained from (13) is only a special group invariant solutions. Next, in order to obtain more general solutions of equation (1), we generalize the residual symmetry method.

In reference [26], Lou start from the truncated Painlevé expansion to propose the definition of consistent Riccati expansion (CRE) solvable. Inspired by this reference, we consider the special case of CRE-consistent Tanh expansion (CTE), which is a more generalized but much simpler method to find interaction solutions between solitons and other nonlinear excitations, such as soliton-resonant solutions, soliton and condial wave, and soliton and sin-cosine wave [38-40].

\section{Consistent Tanh Expansion Solvability of the Higher-Order Broer-Kaup System}

In order to derive the interaction solution of the higher-order Broer-Kaup system (1) by the consistent tanh expansion method, we need to discuss the consistent tanh expansion solvability of the system (1). The first step is to make the leading analysis of the system (1), by balancing the highest order of tanh $(w)$, and one can obtain the following truncated tanh function expansions

$$
\begin{gathered}
\bar{u}=\bar{u}_{0}+\bar{u}_{1} \tanh (w)+w_{x}, \\
\bar{v}=\bar{v}_{0}+\bar{v}_{1} \tanh (w)+\bar{v}_{2} \tanh ^{2}(w) .
\end{gathered}
$$

For convenience later, in equation (16), we substitute $\bar{u}_{0}$ for $\bar{u}_{0}+w_{x}$ (see (19), (20) where $\bar{u}_{0}$ equation has been decoupled). By vanishing all the coefficients of the like powers of tanh $(w)$ after substituting (16) into (1), we obtain eleven overdetermined equations for only six unknown functions $\bar{u}_{0}, \bar{u}_{1}, \bar{v}_{0}, \bar{v}_{1}, \bar{v}_{2}$, and $w$. Vanishing the coefficients of $\tanh ^{4}(w), \tanh ^{5}(w)$ leads to

$$
\bar{u}_{1}=w_{x}, \bar{v}_{2}=-w_{x}^{2}, \text { and } \bar{u}_{1}=2 w_{x}, \bar{v}_{2}=-2 w_{x}^{2},
$$

which have to be considered as two possible case separately. To proceed, we consider the first case.

Case 6. Principle branch. $\bar{u}_{1}=w_{x}, \bar{v}_{2}=-w_{x}^{2}$.

In this case, by substituting the expression $\left\{\bar{u}_{1}=w_{x}, \bar{v}_{2}=-w_{x}^{2}\right\}$ into the remained nine equations, one 
can find that the variables $\bar{u}_{0}, \bar{v}_{0}, \bar{v}_{1}$, and $w$ are consistent and the concert form reading

$$
\bar{v}_{1}=w_{x x}, \bar{v}_{0}=\bar{u}_{0, x}+w_{x}^{2}+w_{x x}
$$

with $\bar{u}_{0}, w$ satisfying the following two equations

$$
\begin{aligned}
& \text { PSTO }:=w_{t}+4\left(w_{x x}+3 w_{x}^{2}+3 \bar{u}_{0} w_{x}\right)_{x} \\
& +16 w_{x}^{3}+12 \bar{u}_{0} w_{x}\left(\bar{u}_{0}+2 w_{x}\right)=0, \\
& \text { STO }:=\bar{u}_{0, t}+4\left(\bar{u}_{0, x x}+3 \bar{u}_{0} \bar{u}_{0, x}+\bar{u}_{0}^{3}\right)_{x}=0 .
\end{aligned}
$$

From (20), we found that $w_{x}$ has been separated out from $\bar{u}_{0}$ so that the $\bar{u}_{0}$ equation is independent $w$, and $w$ equation (19) can also be linearized because it is the potential form of the variable coefficient STO (Sharma-Tasso-Olver) equation-that is PSTO. Then, we have the consistent tanh expansion solvable theorem for the system (1).

Theorem 7. The higher-order Broer-Kaup system (1) is consistent tanh expansion solvable with the consistent tanh expansion as follows:

$$
\begin{aligned}
& u=\bar{u}_{0}+w_{x}(1+\tanh (w)), \\
& v=\bar{u}_{0, x}+w_{x x}(1+\tanh (w))+w_{x}^{2}\left(1-\tanh ^{2}(w)\right),
\end{aligned}
$$

and the $\bar{u}_{0}, w$ satisfies equations (19) and (20).

Case 8. Auxiliary branch. $\bar{u}_{1}=2 w_{x}, \bar{v}_{2}=-2 w_{x}^{2}$.

For this case, it is easy to find that the four variables $\bar{v}_{1}$, $\bar{v}_{0}, \bar{u}_{0}$, and $w$ are not consistent, and the concrete forms of $\bar{u}, \bar{v}$ are

$$
\begin{aligned}
& \bar{u}=w_{x}(1+2 \tanh (w))+\frac{w_{x x}}{w_{x}}, \\
& \bar{v}=\frac{w_{x x}^{2}}{w_{x}^{2}}-\frac{w_{x x x}}{w_{x}}+2 w_{x x} \tanh (w)+2 w_{x}^{2}\left(1-\tanh ^{2}(w)\right),
\end{aligned}
$$

while $w$ satisfies the following equations:

$$
\begin{gathered}
w_{t}+4\left(4 w_{x}^{3}-2 w_{x x x}+3 \frac{w_{x x}^{2}}{w_{x}}\right)=0, \\
\left(4 w_{x}^{2}-\partial_{x}^{2}\right)\left(\frac{w_{x x x}}{w_{x}}-\frac{3}{2} \frac{w_{x x}^{2}}{w_{x}^{2}}\right)+10 w_{x x}^{2}=0 .
\end{gathered}
$$

It is not difficult to check that this case is not a consistent tanh expansion solvable. However, it is still a useful way to construct the special type of exact solution to the higherorder Broer-Kaup system (1) by solving the overdetermined equations (23).

\section{Explict Solution to the Higher-Order Broer- Kaup System}

The explict solutions of nonlinear PDE play an important role in nonlinear science and engineering, and there are many kinds of effective methods that have been established to construct explict solutions for nonlinear equations, such as symmetry reductions [30, 31], bilinear method [41-43], Darboux transformation [44], and Painlevé analysis [45], but most of them are more difficult to find interaction solutions which are an important and meaningful research topic [46-48]. Fortunately, the above consistent tanh expansion (CTE) method can be easily applied to investigate interaction solutions between a soliton and any other types of nonlinear excitations. Next, we construct the interaction solutions to the HBK system on the basis of CTE solvable Theorem 7.

According to Theorem 7, we find that the explicit solutions to HBK system can be obtained by solving the $w$ and $u_{0}$ equation (19), (20). In order to solve the $w$ equation (19), if we choose any fixed solution to the STO equation (20), we will get the solution of $w$ by solving the variable coefficient PSTO equation (19).

Now, we first restrict the simplest solution $u_{0}=c$ to equation (20) and substitute it into equation (19), which will be simplified in the following constant coefficient PSTO equation

$$
w_{t}+4\left(w_{x x}+3 w_{x}^{2}+3 c w_{x}\right)_{x}+16 w_{x}^{3}+12 c w_{x}\left(c+2 w_{x}\right)=0
$$

From equation (24), we know that $w$ has the following trivial solution,

$$
w=k x+q t+z_{0}, \text { and } q=-4\left(3 c^{2} k+6 c k^{2}+4 k^{3}\right),
$$

where $z_{0}$ is an arbitrary constant, and the corresponding single soliton solution to the HBK system is as follows

$$
\begin{aligned}
& u=k \tanh \left(k x+q t+z_{0}\right)+k+c, \\
& v=k^{2} \operatorname{sech}^{2}\left(k x+q t+z_{0}\right) .
\end{aligned}
$$

In order to study the properties of the solution, we plot the structure of the solution. Figures 1(a) and 1(b) display this kind of single soliton solution for the fields $u$ and $v$.

In what follows, we are interesting in finding another kind of solution in the form

$$
w=k x+q t+\frac{p}{2}
$$

which reflects the interaction solution between a solition and an STO/PSTO wave. Substituting (27) into equation (24) will lead to

$$
p_{t}+4 p_{x}^{3}+4\left(\frac{3}{2} p_{x}^{2}+3 c_{1} p_{x}+3 c_{1}^{2} p+p_{x x}\right)_{x}+12 c_{1} p_{x}^{2}=0
$$




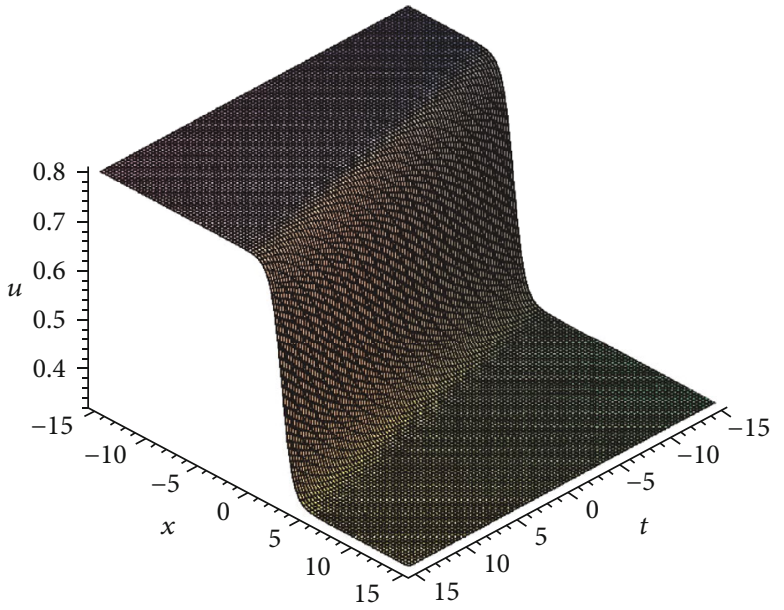

(a)

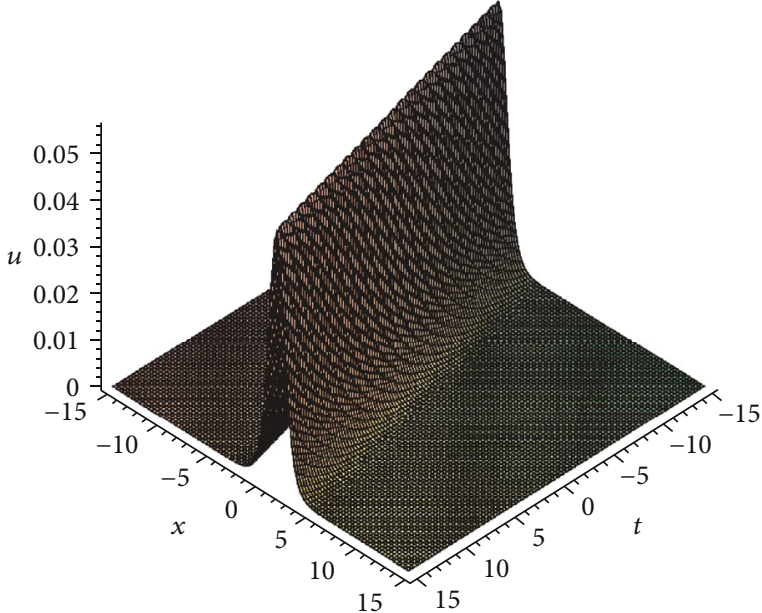

(b)

Figure 1: Plot (a) refers to the single kink solutions of $u$, and (b) refers to bell solitary wave of $v$ in equation (26), with parameters: $\left\{c=4 / 5, k=-4 / 17, z_{0}=2\right\}$.

with $c_{1}=c+2 k$. The corresponding solution to the HBK system (1) is as follows:

$$
\begin{aligned}
& u=\frac{1}{2}\left(2 k+p_{x}\right)(1+\tanh (w))+c \\
& v=\left(k+\frac{1}{2} p_{x}\right)^{2} \operatorname{sech}^{2}(w)+\frac{1}{2} p_{x x}(1+\tanh (w)) .
\end{aligned}
$$

Now, employing the known solutions to (28), such as resonant soliton solutions and multisoliton solutions from literature [49-52], we will construct the interaction solutions between a soliton and STO/PSTO wave.

4.1. The Resonant Multisoliton Solutions. It can be verified that equation (28) possesses the following multiple wave solution

$$
p=\ln \left(\sum _ { i = 1 } ^ { n } \left(c_{i} \exp \left(k_{i} x-4\left(k_{i}^{3}+3 k_{i} c_{1}^{2}+3 c_{1} k_{i}^{2}\right) t+z_{i}\right)\right.\right.
$$

Substituting (27) and (30) into (29), the $(n+1)$ resonant multisoliton solutions to the HBK system can be directly obtained, which displays soliton fission and fusion. For example, if we take $n=2$ in (30), with parameters: $\left\{c_{1}=-1 / 2, g_{1}\right.$ $\left.=1 / 15, k_{1}=1, z_{1}=1, g_{2}=1 / 12, k_{2}=-1 / 13, z_{2}=2\right\}$ and $k>0$ , then the solution (29) shows the two solitary wave fusion. It shows that two solitons fuse into one soliton after collide, which reflects the inelastic collision between solitons and is displayed in Figure 2.

And when the $k<0$, the solution (29) shows the soliton fission, which also reflects the inelastic collision between solitons and is displayed in Figure 3.

If we choose $n=3$ in (30), as Figure 4, we derive the three kink solitary wave fusion with parameters: $\left\{c_{1}=-2 / 3, g_{1}=\right.$
$2, g_{2}=10, g_{3}=3 / 5, k_{1}=1.2, k_{2}=5 / 6, k_{3}=1 / 3, z_{1}=3.8, z_{2}=$ $\left.3 / 4, z_{3}=3\right\}$.

\subsection{Soliton Interactions with Periodic Waves}

4.2.1. Soliton Interactions with Sine-Cosine Periodic Waves. It is not difficult to verify that equation (28) possesses the following explicit solutions

$$
\begin{aligned}
p= & \ln \left\{\sum_{i=1}^{n} a_{i} \cos \left\{k_{i}\left(-x+4\left(3 l_{i}^{2}-k_{i}^{2}+3\left(c_{1}+l_{i}\right)^{2}\right) t\right)\right\} \exp \right. \\
& \cdot\left\{l_{1} x-4 t\left(l_{i}^{3}+3\left(l_{i}\left(c_{1}^{2}-k_{i}^{2}\right)-c_{1}\left(k_{i}^{2}-2 l_{i}^{2}\right)\right)\right\}\right\},
\end{aligned}
$$

where $a_{i}, k_{i}, l_{i}$ denote arbitrary constants. Substituting the equation (31) into (29), we can obtain soliton interactions with sine-cosine periodic waves.

4.2.2. Soliton Interactions with Cnoidal Periodic Waves. Interaction solution between soliton and cnoidal periodic waves can display many more interesting physical phenomena, and it can be difficult to obtained by other methods. Here, we take $p$ as the following special Jacobian elliptic function

$$
p=l_{1} x+l_{2} t+\mu \mathrm{E}_{\pi}\left(\operatorname{sn}\left(k_{1} x+k_{2} t, m\right), n, m\right),
$$

$l_{1}, l_{2}, k_{1}, k_{2}, \mu$, and $m$ are determined later, where $\operatorname{s} n(z, m)$ is the usual Jacobian elliptic sine function and

$$
E_{\pi}(\zeta, n, m)=\int_{0}^{\zeta} \frac{d t}{\left(1-n t^{2}\right) \sqrt{\left(1-t^{2}\right)\left(1-m^{2} t^{2}\right)}}
$$




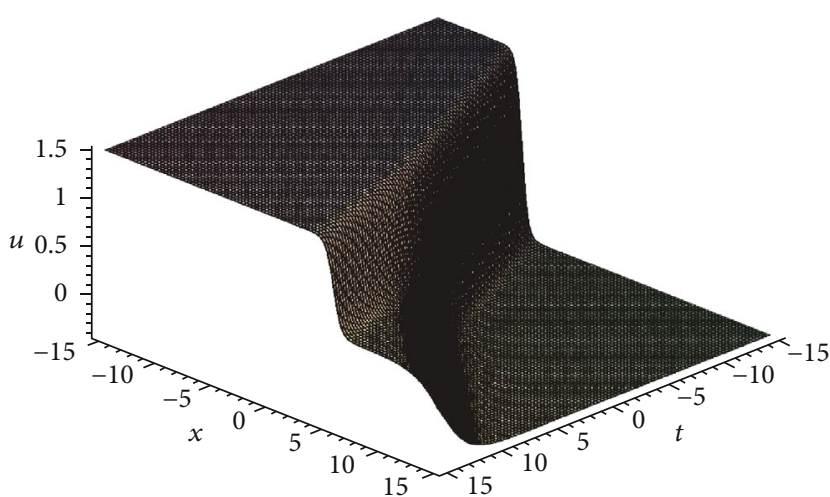

(a)

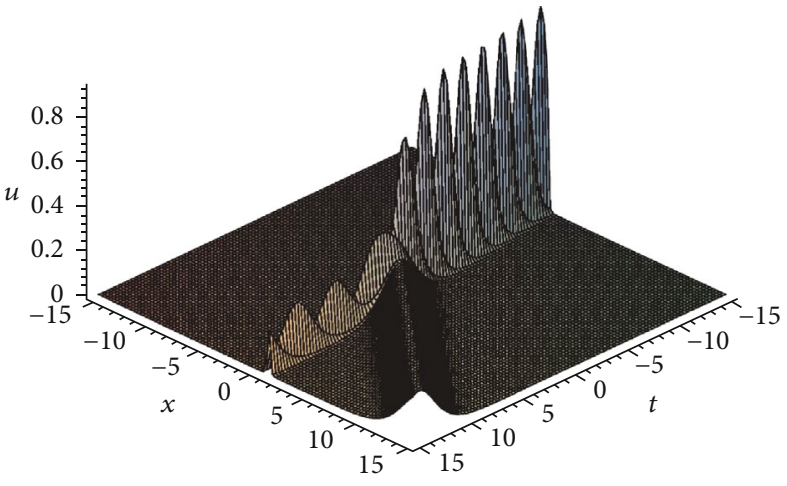

(b)

Figure 2: Plot (a) and (b) to the resonant soliton solution of $u$ and $v$ with $k>0$. (a) is a graph of $u$, which shows that two kink solitary waves collide and fuse into a resonant soliton solution at $t=0$. (b) is a graph of $v$, which shows that two bell solitary waves collide and fuse into a resonant soliton solution at $t=0$.

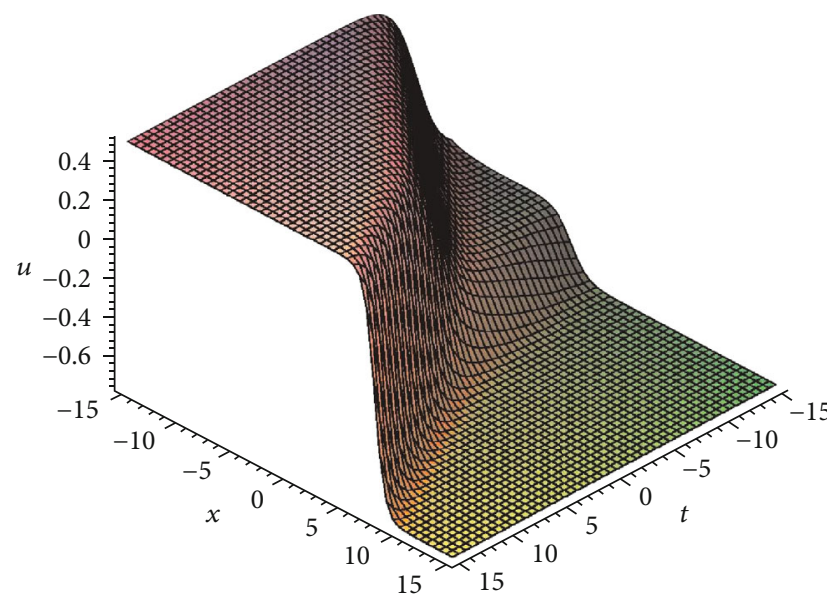

(a)

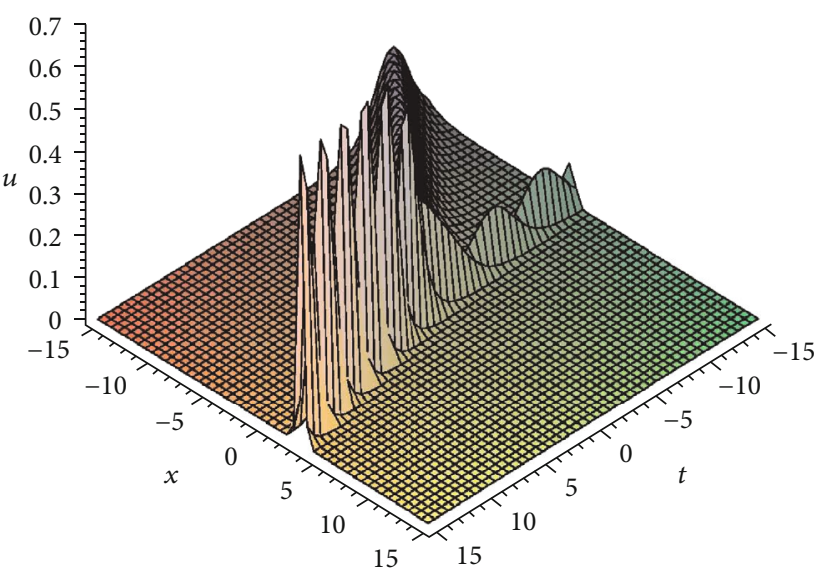

(b)

Figure 3: Plot (a) and (b) to the resonant soliton solution of $u$ and $v$ with $k<0$. (a) is the two kink solitary wave fission solution for $u$, and (b) is the solitary wave fission for $v$.

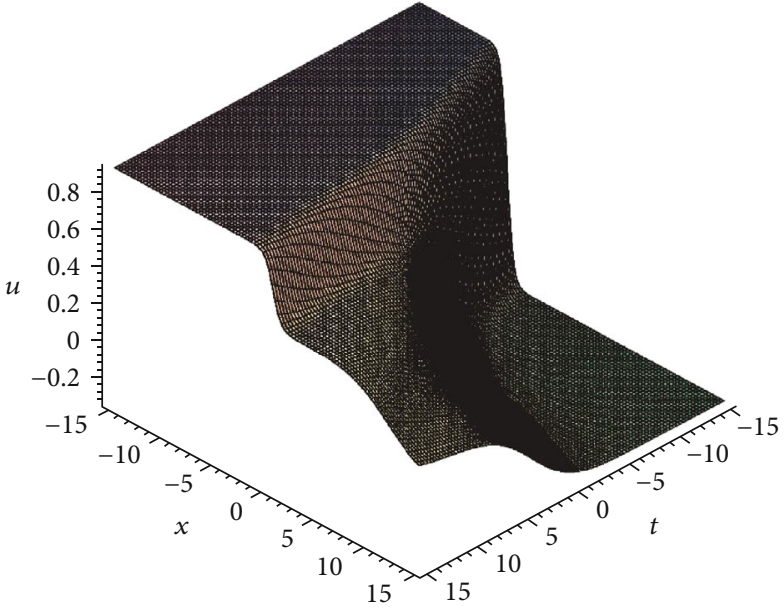

(a)

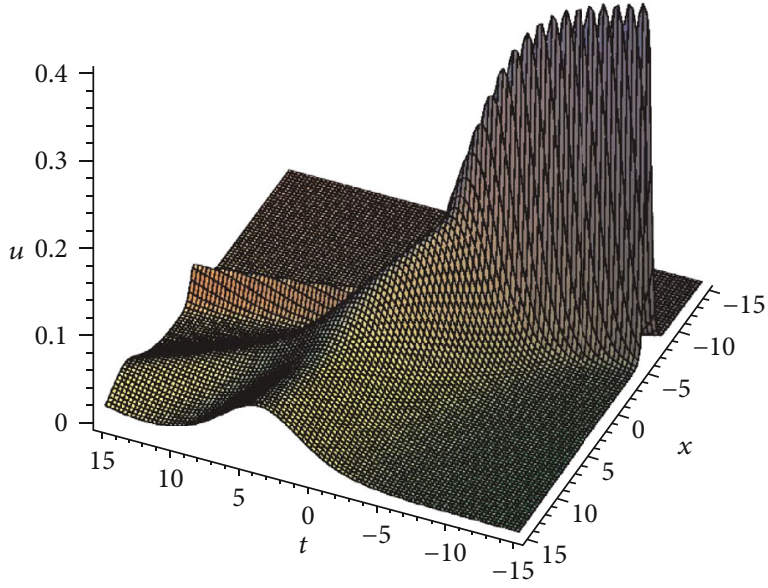

(b)

Figure 4: Plot (a) and (b) to the resonant soliton solution of $u$ and $v$. (a) is a graph of $u$, which shows that three kink solitary waves collide and fuse into a resonant soliton solution at $t=0$. (b) is a graph of $v$, which shows that three bell solitary waves collide and fuse into a resonant soliton solution at $t=0$. 


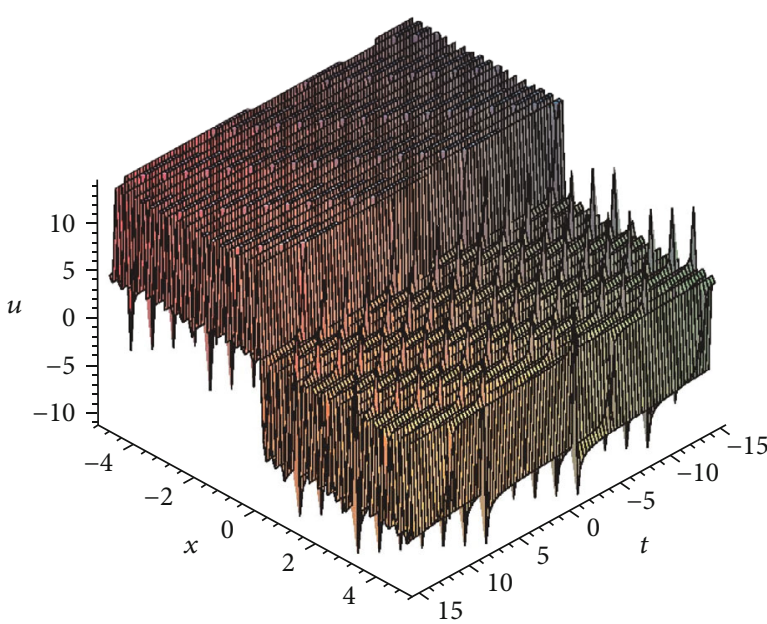

(a)

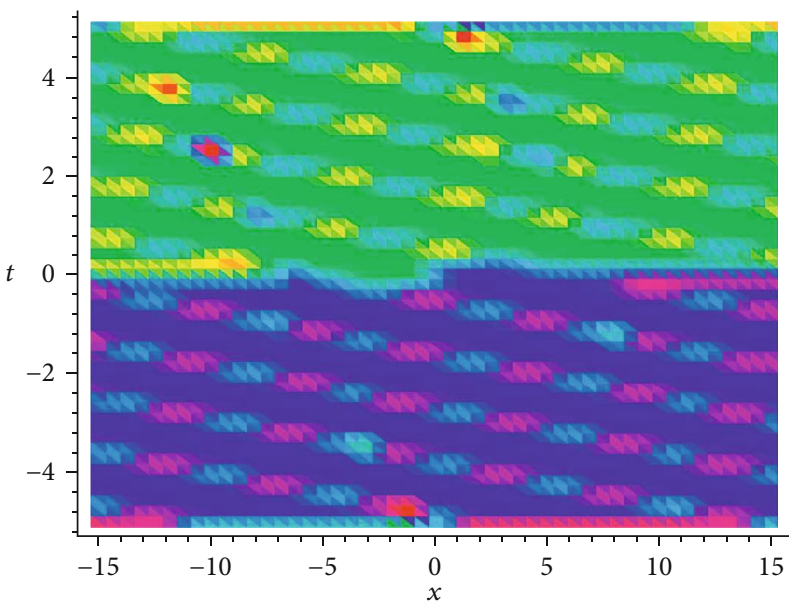

(c)

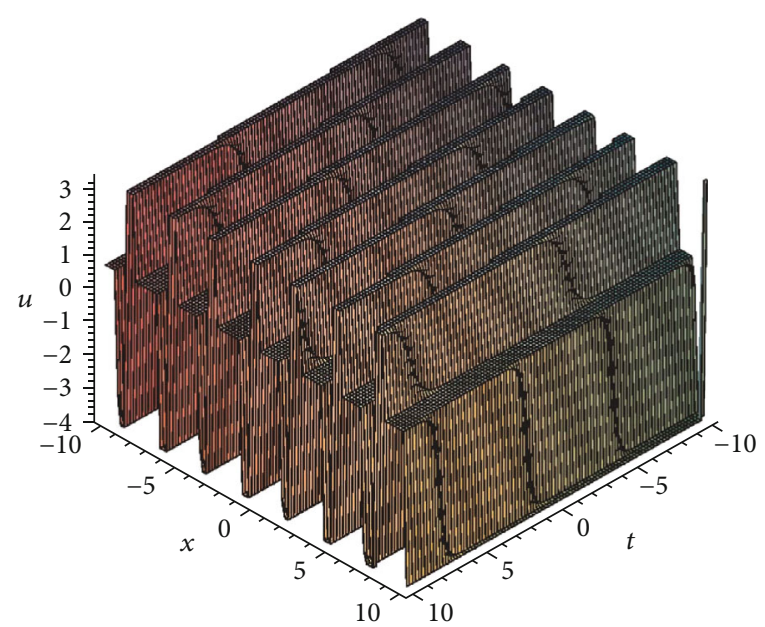

(b)

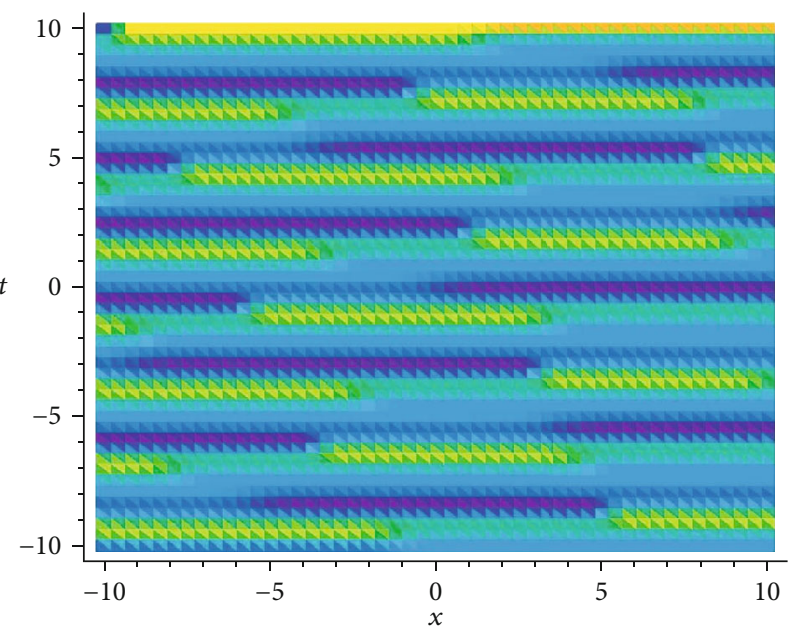

(d)

Figure 5: (a) The interaction solution between soliton and sine-cosine wave of $u$ in equation (29) by substituting (27) and (31), with parameters: $\left\{a_{1}=1 / 3, a_{2}=1 / 3, c_{1}=-6, k_{1}=1 / 4, k_{2}=1 / 4, l_{1}=3 / 20, l_{2}=3 / 20\right\}$. (b) The interaction solution between soliton-cnoidal wave of $u$ in equation (29) by substituting (27) and (32), with parameters: $\left\{\mu=50, k_{1}=1 / 4, l_{1}=-1, l_{2}=0.37, c_{1}=2 / 3, m=0.7\right\}$. (c) and (d) are the overhead view of the wave (a) and (b).This interaction solution reflects the elastic collision.

is the third type of incomplete elliptic integral. Substituting (32) into (28) and solving the overdetermined equations with the help of maple will come the following result

$$
\begin{aligned}
k_{2}= & -\frac{1}{\mu}\left(4 k_{1}^{3} \mu^{3}+12 c_{1} k_{1}^{2} \mu^{2}+12 k_{1}^{2} l_{1} \mu^{2}+1_{2} c_{1}^{2} k_{1} \mu\right. \\
& \left.+24 c_{1} k_{1} l_{1} \mu+1_{2} k_{1} l_{1}^{2} \mu+1_{2} c_{1}^{2} l_{1}+1_{2} c_{1} l_{1}^{2}+4 l_{1}^{3}+l_{2}\right),
\end{aligned}
$$

with $n=0$, and $l_{1}, l_{2}, k_{1}, m, \mu$ are arbitrary constants. Substituting equation (32) and (34) into (29), we can obtain soliton interactions with cnoidal periodic waves. It is concluded that the CTE solutions (29) present the interaction solutions to multiple solitons and multiple periodic waves by substituting (31), (32), and (34). Here, we plot the structure of the solution, and Figures 5(a)-5(d) display the two kinds of interactions solution for the field $u$.
In a word, according to equations (19) and (20), taking any solution $u_{0}$ to the STO equation and the variable coefficient PSTO system, one can get the corresponding interaction solutions to the HBK system (1) via the theorem 7 .

For the non-CTE case, we can still find some special exact solutions for the overdetermined system (23), such as the system (23) possessing the following exact solution

$$
w_{1}=\frac{1}{2} \ln \frac{s_{1} x+s_{2}-4}{s_{1} x+s_{2}+4}+s_{3},
$$

$w_{2}=-\frac{1}{2} \ln 2+\frac{1}{4} \ln \frac{(\tan (\sqrt{2} / 8)(2 x+t+1)+1)^{2}}{(\tan (\sqrt{2} / 8)(2 x+t+1)+1-8 \sqrt{2})^{2}}$ 
Substituting the expressions (35) and (36) into (22) yields the special rational solutions to a single soliton solution, respectively.

\section{Summary and Discussion}

It is summarized that the residual symmetry of the higherorder Broer-Kaup (HBK) system is obtained from the standard truncated Painlevé expansion. For the original nonlinear system, the residual symmetries are nonlocal. However, it can be localized to Lie point symmetries by introducing a properly auxiliary dependent variable, and the finite transformation of the residual symmetry is obtained by solving the standard Lie's initial value problem, and the group invariant solutions of HBK are derived. Furthermore, in order to study other types of solutions for the HBK system, we did not employ the general symmetry reduction method, but use the CTE method, by discussing the consistent tanh expansion solvable of the HBK system, we derive abundant exact solutions to the HBK system, and these solutions reveal the interaction among solitons and other types of nonlinear excitations especially the STO/PSTO wave such as the multiple resonant solitons and periodic waves. These kinds of solution can be easily applicable to the analysis of physically interesting processes.

The method presented in this paper would be applied to other various interesting integrable models. One can also consider the relationship between residual symmetry and other nonlocal symmetries. And more research should be done on the CTE method and more types of the interaction solutions.

\section{Appendix}

$$
\begin{aligned}
&\left(\frac{1}{\phi}\right)^{0}: u_{0 t}+4 u_{0 x x x}+24 u_{0} v_{0 x}+24 u_{0 x} v_{0}-12 u_{0 x}^{2} \\
&-12 u_{0} u_{0 x x}+12 u_{0}^{2} u_{0 x}=0 \\
&\left(\frac{1}{\phi}\right)^{1}: u_{1 t}+4 u_{1 x x x}+24 u_{1} v_{0 x}+24 u_{0} v_{1 x}+24 u_{1 x} v_{0}+24 u_{0 x} v_{1} \\
&-12 u_{1} u_{0 x x}-12 u_{0} u_{1 x x}+24 u_{0} u_{0 x} u_{1}+12 u_{0}^{2} u_{1 x}=0
\end{aligned}
$$

$$
\begin{aligned}
\left(\frac{1}{\phi}\right)^{2}: & -12 u_{0}^{2} u_{2} \phi_{x}-24 u_{0} v_{1} \phi_{x}+24 u_{0} \phi_{x} u_{1 x} \\
& +24 u_{0} u_{1} u_{1 x}+12 u_{0} u_{1} \phi_{x x}-24 v_{0} u_{1} \phi_{x} \\
& +24 u_{0 x} \phi_{x} u_{1}+12 u_{0 x} u_{1}^{2}+24 u_{0} v_{2 x}+24 u_{0 x} v_{2} \\
& +24 v_{1 x} u_{1}+24 v_{1} u_{1 x}-u_{1} \phi_{t}-12 u_{1 x x} \phi_{x} \\
& -12 u_{1 x}^{2}-12 u_{1 x x} u_{1}-12 u_{1 x} \phi_{x x}-4 u_{1} \phi_{x x x}=0,
\end{aligned}
$$

$$
\begin{aligned}
\left(\frac{1}{\phi}\right)^{3}: & -24 u_{0} u_{1} \phi_{x}^{2}-24 u_{0} \phi_{x} u_{1}^{2}-48 u_{0} v_{2} \phi_{x}-48 v_{1} u_{1} \phi_{x} \\
& +24 u_{1 x} \phi_{x}^{2}+48 \phi_{x} u_{1 x x}+24 u_{1} \phi_{x} \phi_{x x}+12 u_{1 x} u_{1}^{2} \\
& +12 \phi_{x x} u_{1}^{2}+24 u_{1} v_{2 x}+24 v_{2} u_{1 x}=0
\end{aligned}
$$

$$
\left(\frac{1}{\phi}\right)^{4}: 2 \phi_{x}^{2}+3 u_{1} \phi_{x}+u_{1}^{2}+6 v_{2}=0
$$

$$
\begin{aligned}
\left(\frac{1}{\phi}\right)^{0}: v_{0 t} & +4 v_{0 x x x}+12 u_{0 x} v_{0 x}+12 u_{0} v_{0 x x}+24 u_{0} v_{0} u_{0 x} \\
& +12 u_{0}^{2} v_{0 x}+24 v_{0} v_{0 x}=0
\end{aligned}
$$

$$
\begin{aligned}
\left(\frac{1}{\phi}\right)^{1}: v_{1 t} & +4 v_{1 x x x}+12 u_{1 x} v_{0 x}+12 u_{0 x} v_{1 x}+12 u_{1} v_{0 x x} \\
& +12 u_{0} v_{1 x x}+24 u_{1} v_{0} u_{0 x}+24 u_{0} v_{1} u_{0 x} \\
& +24 u_{0} v_{0} u_{1 x}+12 u_{0}^{2} v_{1 x}+24 u_{0} u_{1} v_{0 x} \\
& +24 v_{1} v_{0 x}+24 v_{0} v_{1 x}=0
\end{aligned}
$$

$$
\begin{aligned}
\left(\frac{1}{\phi}\right)^{2}: & -12 u_{1} v_{0 x} \phi_{x}-12 u_{0}^{2} v_{1} \phi_{x}+24 u_{0} v_{2} u_{0 x}-24 u_{0} v_{1 x} \phi_{x} \\
& +24 u_{0} v_{1 x} u_{1}+24 u_{0} v_{1} u_{1 x}-12 u_{0} v_{1} \phi_{x x} \\
& -24 v_{0} v_{1} \phi_{x}+24 v_{0} u_{1} u_{1 x}-12 u_{0 x} v_{1} \phi_{x}+24 u_{0 x} v_{1} u_{1} \\
& -24 u_{0} v_{0} u_{1} \phi_{x}+12 u_{0 x} v_{2 x}+24 v_{0 x} v_{2}-12 v_{1 x x} \phi_{x} \\
& +12 u_{1} v_{1 x x}+24 v_{1} v_{1 x}+12 v_{1 x} u_{1 x}-12 v_{1 x} \phi_{x x} \\
& -v_{1} \phi_{t}-4 v_{1} \phi_{x x x}+12 u_{0}^{2} v_{2 x}+12 v_{0 x} u_{1}^{2}+12 u_{0} v_{2 x x} \\
& +24 v_{0} v_{2 x}+v_{2 t}+4 v_{2 x x x}=0,
\end{aligned}
$$

$$
\begin{aligned}
\left(\frac{1}{\phi}\right)^{3}: 24 u_{0} v_{1} \phi_{x}^{2} & -24 u_{0}^{2} v_{2} \phi_{x}-24 v_{0} u_{1}^{2} \phi_{x}-48 u_{0} v_{2 x} \phi_{x} \\
& +24 u_{0} u_{1} v_{2 x}+24 u_{0} v_{2} u_{1 x}-24 u_{0} v_{2} \phi_{x x} \\
& -48 v_{0} v_{2} \phi_{x}-24 u_{0 x} v_{2} \phi_{x}+24 u_{0 x} v_{2} u_{1} \\
& -36 u_{1} v_{1 x} \phi_{x}-12 v_{1} u_{1 x} \phi_{x}+24 v_{1} \phi_{x} \phi_{x x} \\
& +24 v_{1} u_{1} u_{1 x}-12 u_{1} v_{1} \phi_{x x}-48 u_{0} u_{1} v_{1} \phi_{x} \\
& -2 v_{2} \phi_{t}+24 v_{1 x} \phi_{x}^{2}+12 u_{1}^{2} v_{1 x}-24 v_{1}^{2} \phi_{x} \\
& -24 v_{2 x x} \phi_{x}+12 u_{1} v_{2 x x}+24 v_{1} v_{2 x} \\
& +12 u_{1 x} v_{2 x}-24 v_{2 x} \phi_{x x}+24 v_{2} v_{1 x} \\
& -8 v_{2} \phi_{x x x}=0
\end{aligned}
$$

$$
\begin{aligned}
\left(\frac{1}{\phi}\right)^{4}: 72 u_{0} v_{2} \phi_{x}^{2} & -72 u_{0} v_{2} u_{1} \phi_{x}-24 v_{1} \phi_{x}^{3}+36 v_{1} u_{1} \phi_{x}^{2} \\
& -36 v_{1} u_{1}^{2} \phi_{x}+72 v_{2 x} \phi_{x}^{2}-60 v_{2 x} \phi_{x} u_{1} \\
& +12 v_{2 x} u_{1}^{2}-72 v_{2} v_{1} \phi_{x}-24 v_{2} \phi_{x} u_{1 x} \\
& +72 v_{2} \phi_{x} \phi_{x x}+24 v_{2} u_{1} u_{1 x}-24 v_{2} u_{1} \phi_{x x} \\
& +24 v_{2 x x}=0,\left(\frac{1}{\phi}\right)^{5}: v_{2} \\
& +u_{1}^{2}-2 u_{1} \phi_{x}+2 \phi_{x}^{2}=0
\end{aligned}
$$

\section{Data Availability}

The [exact solutions] data used to support the findings of this study are included within the article. 


\section{Conflicts of Interest}

The authors declare that there are no conflicts of interest regarding the publication of this paper.

\section{Acknowledgments}

The project is supported by the National Natural Science Foundation of China (Grant Nos. 12001424, 11471004, and 11775047), the Chinese Post Doctoral Science Foundation (No. 2020M673332), the Natural Science Basic Research Program of Shaanxi Province (No. 2021JZ-21), and Liaocheng University Level Science and Technology Research Fund (No. 318012018).

\section{References}

[1] P. J. Olver, Applications of Lie Groups to Differential Equations, Springer, Berlin, 1986.

[2] G. W. Bluman and S. Kumei, Symmetries and differential equations, Springer-Verlag, New York, 1989.

[3] J. Shi, M. Zhou, and H. Fang, "Group-invariant solutions, nongroup-invariant solutions and conservation laws of Qiao equation," Journal of Applied Analysis Computation, vol. 9, no. 5, pp. 2023-2036, 2019.

[4] C. Qin, S. Tian, L. Zou, and T. Zhang, "Lie symmetry analysis, conservation laws and exact solutions of fourth-order time fractional burgers equation," Journal of Applied Analysis Computation, vol. 8, no. 6, pp. 1727-1746, 2018.

[5] V. Kumar, L. Kaur, A. Kumar, and M. E. Koksal, "Lie symmetry based-analytical and numerical approach for modified Burgers-KdV equation," Results in physics, vol. 8, pp. 1136$1142,2018$.

[6] L. Kaur and R. K. Gupta, "Kawahara equation and modified Kawahara equation with time dependent coefficients: symmetry analysis and generalized G'G-expansion method," Mathematical Methods in the Applied Sciences, vol. 36, no. 5, pp. 584-600, 2018.

[7] A. M. Wazwaz and L. Kaur, "Complex simplified Hirota's forms and Lie symmetry analysis for multiple real and complex soliton solutions of the modified KdV-Sine-Gordon equation," Nonlinear Dynamics, vol. 95, no. 3, pp. 22092215, 2019.

[8] J. J. Mao, S. F. Tian, T. T. Zhang, and X. J. Yan, "Lie symmetry analysis, conservation laws and analyticalsolutions for chiral nonlinear Schrödinger equationin $(2+1)$-dimensions," Nonlinear Analysis: Modelling and Control, vol. 25, no. 3, pp. 358-377, 2020.

[9] G. A. Guthrie, "Recursion operators and non-local symmetries," Proceedings of the Royal Society of London. Series A, vol. 446, pp. 107-114, 1994.

[10] S. Lou, "Symmetries of the KdV equation and four hierarchies of the integrodifferential KdV equations," Journal of Mathematical Physics, vol. 35, no. 5, pp. 2390-2396, 1994.

[11] S. Lou and X. Hu, "Non-local symmetries via darboux transformations," Journal of Physics A: Mathematical and General, vol. 30, no. 5, pp. L95-L100, 1997.

[12] S. Lou, X. Hu, and Y. Chen, "Nonlocal symmetries related to Bäcklund transformation and their applications," Journal of Physics A-Mathematical and Theoretical, vol. 45, no. 15, pp. 1-14, 2012.
[13] X. Tang and Z. Liang, "Nonlocal symmetries and conservation laws of the Sinh-Gordon equation," Journal of Nonlinear Mathematical Physics, vol. 24, no. 1, pp. 93-106, 2017.

[14] O. I. Morozov and M. V. Pavlov, "Bäcklund transformations between four lax-integrable 3D equations," Journal of Nonlinear Mathematical Physics, vol. 24, no. 4, pp. 465-468, 2017.

[15] M. Euler, N. Euler, and E. G. Reyes, "Multipotentializations and nonlocal symmetries: Kupershmidt, Kaup-Kupershmidt and Sawada-Kotera equations," Journal of Nonlinear Mathematical Physics, vol. 24, no. 3, pp. 303-314, 2017.

[16] S. Lou, "Conformal invariance and integrable models," Journal of Physics A: Mathematical and General, vol. 30, no. 13, pp. 4803-4813, 1997.

[17] S. Lou, "Negative Kadomtsev-Petviashvili hierarchy," Physica Scripta, vol. 57, no. 4, pp. 481-485, 1998.

[18] X. B. Hu, S. Y. Lou, and X. M. Qian, "Nonlocal symmetries for bilinear equations and their Applications," Studies in Applied mathematics, vol. 122, no. 3, pp. 305-324, 2009.

[19] S. Y. Lou, Residual Symmetries and Bäcklund Transformations, Physics, 2013.

[20] X. Gao, S. Lou, and X. Tang, "Bosonization, singularity analysis, nonlocal symmetry reductions and exact solutions of supersymmetric KdV equation," Journal of High Energy Physics, vol. 5, pp. 1-29, 2013.

[21] Y. Xia, X. Xin, and S. Zhang, "Residual symmetry, interaction solutions and conservation laws of the $(2+1)$ dimensional dispersive long-wave system," Chinese Physics B, vol. 26, no. 3, article 030202, 2017.

[22] M. Dong, S. Tian, X. Yan, and T. Zhang, "Nonlocal symmetries, conservation laws and interaction solutions for the classical Boussinesq-Burgers equation," Nonlinear Dynamics, vol. 95, no. 1, pp. 273-291, 2019.

[23] H. Wang and Y. Zhang, "Residual symmetries and Bäcklund transformations of $(2+1)$-dimensional strongly coupled Burgers system," Advances in Mathematical Physics, vol. 2020, Article ID 6821690, 8 pages, 2020.

[24] Q. Miao, X. Xin, and Y. Chen, "Nonlocal symmetries and explicit solutions of the AKNS system," Applied in Mathematics Letters, vol. 28, no. 2, pp. 7-13, 2014.

[25] X. Xin, Y. Liu, and X. Liu, "Nonlocal symmetries, exact solutions and conservation laws of the coupled Hirota equations," Applied Mathematics Letters, vol. 55, pp. 63-71, 2016.

[26] S. Lou, "Consistent Riccati expansion for integrable systems," Studies in Applied Mathematics, vol. 134, no. 3, pp. 372-402, 2015.

[27] C. Chen and S. Lou, "CTE solvability, nonlocal symmetries and exact solutions of dispersive water wave system," Communications in Theoretical Physics, vol. 61, no. 5, pp. 545-550, 2014.

[28] J. Chen, H. Wu, and Q. Zhu, "Bäcklund transformation and soliton-cnoidal wave interaction solution for the coupled Klein-Gordon equations," Nonlinear Dynamics, vol. 91, no. 3, pp. 1949-1961, 2018.

[29] J. Chen and S. Zhu, "Residual symmetries and soliton-cnoidal wave interaction solutions for the negative-order Korteweg-de Vries equation," Applied Mathematics Letters, vol. 73, pp. 136142, 2017.

[30] L. Huang and Y. Chen, "Nonlocal symmetry and similarity reductions for the Drinfeld-Sokolov-Satsuma- Hirota system," Applied Mathematics Letters, vol. 64, pp. 177-184, 2017. 
[31] Y. Yue, L. Huang, and Y. Chen, "Localized waves and interaction solutions to an extended (3+1)-dimensional Jimbo-Miwa equation," Applied Mathematics Letters, vol. 89, pp. 70-77, 2019.

[32] P. Liu, H. R. Xu, and J. R. Yang, "The Boussinesq equation: Lax pair, Bäcklund transformation, symmetry group transformation and consistent Riccati expansion solvability," Acta Physica Sinica, vol. 69, no. 1, article 010203, 2020.

[33] S. Lou and X. Hu, "Infinitely many lax pairs and symmetry constraints of the KP equation," Journal of Mathematics and Physics, vol. 38, no. 12, pp. 6401-6427, 1997.

[34] E. Fan, "Solving Kadomtsev-Petviashvili equation via a new decomposition and Darboux transformation," Communications in Theoretical Physics, vol. 37, no. 2, pp. 145-148, 2002.

[35] D. Huang, D. S. Li, and H. Q. Zhang, "Explicit N-fold Darboux transformation and multi-soliton solutions for the $(1+1)$ dimensional higher-order Broer-Kaup system," Chaos, Solitons \& Fractals, vol. 33, no. 5, pp. 1677-1685, 2007.

[36] X. N. Li, G. M. Wei, Y. P. Liu, Y. Q. Liang, and X. H. Meng, "Painlevé analysis and new analytic solutions for $(1+1)$ dimensional higher-order Broer Kaup system with symbolic computation," International Journal of Modern Physics B, vol. 28, no. 14, p. 1450067, 2014.

[37] X. Xin and X. Liu, "Interaction solutions for (1+1)-dimensional higher-order Broer-Kaup system," Communications in Theoretical Physics, vol. 66, no. 5, pp. 479-482, 2016.

[38] B. Ren, J. Lin, and Z.-M. Lou, "Consistent Riccati expansion and rational solutions of the Drinfel'd-Sokolov- Wilson equation," Applied Mathematics Letters, vol. 105, article 106326, 2020.

[39] B. Ren, J. Lin, and P. Liu, "Soliton molecules and the CRE method in the extended mKdV equation," Communications in Theoretical Physics, vol. 72, no. 5, article 055005, 2020.

[40] X. W. Jin and J. Lin, "Rogue wave, interaction solutions to the KMM system," Journal of Magnetism and Magnetic Materials, vol. 502, article 166590, 2020.

[41] W. Ma, "A search for lump solutions to a combined fourthorder nonlinear PDE in (2+1)-dimensions," Journal of Applied Analysis and Computation, vol. 9, no. 4, pp. 1319-1332, 2019.

[42] L. Zhang, W. X. Ma, and Y. Huang, "Lump solutions of a nonlinear PDE combining with a new fourth-order term," Advances in Mathematical Physics, vol. 2020, Article ID 3542320, 8 pages, 2020.

[43] H. Ma, Y. Bai, and A. Deng, "Multiple lump solutions of the ()-dimensional Fokas equation," Advances in Mathematical Physics, vol. 2020, Article ID 3407676, 7 pages, 2020.

[44] X. Xin, Y. Xia, H. Liu, and L. Zhang, "Darboux transformation of the variable coefficient nonlocal equation," Journal of Mathematical Analysis and Applications, vol. 490, no. 1, article 124227, 2020.

[45] L. Kaur and A. M. Wazwaz, "Painlevé analysis and invariant solutions of generalized fifth-order nonlinear integrable equation," Nonlinear Dynamics, vol. 94, no. 4, pp. 2469-2477, 2018.

[46] Y. Wang, "CTE method to the interaction solutions of Boussinesq-burgers equations," Applied Mathematics Letters, vol. 38, pp. 100-105, 2014.

[47] X. Wang, S. Bilige, and J. Pang, "Rational solutions and their interaction solutions of the ()-dimensional Jimbo-Miwa equation," Advances in Mathematical Physics, vol. 2020, Article ID 9260986, 18 pages, 2020.
[48] M. Jia, "Symmetry analysis, Bäcklund transformations, and interaction solutions for an $\mathrm{AB}$ modified $\mathrm{KdV}$ system," Advances in Mathematical Physics, vol. 2020, Article ID 4652126, 13 pages, 2020.

[49] S. Wang, X. Y. Tang, and S. Y. Lou, "Soliton fission and fusion: Burgers equation and Sharma-Tasso-Olver equation," Chaos, Solitons and Fractals, vol. 21, no. 1, pp. 231-239, 2004.

[50] Z. J. Lian and S. Y. Lou, "Symmetries and exact solutions of the Sharma-Tass-Olver equation," Nonlinear Analysis, vol. 63, no. 5-7, pp. e1167-e1177, 2005.

[51] J. J. Mao, S. F. Tian, L. Zou, and T. T. Zhang, "Stability analysis, optical solitons and complexitons of the two-dimensional complex Ginzburg-Landau equation," Journal of Electromagnet Wave and Application, vol. 33, no. 9, pp. 1224-1238, 2019.

[52] J. J. Mao, S. F. Tian, L. Zou, T. T. Zhang, and X. J. Yan, “Bilinear formalism, lump solution, lumpoff and instanton/rogue wave solution of a $(3+1)$-dimensional B-type KadomtsevPetviashvili equation," Nonlinear Dynamics, vol. 95, no. 4, pp. 3005-3017, 2019. 